\title{
Recent Development of Automation and IoT in Agriculture
}

\author{
Chidananda Kashyap, Bharath Y Kashyap, Guruprasad K, Shrinivasa D, Prashant Kumar \\ Shrivastava
}

\begin{abstract}
This paper presents a brief overview of the automation and IoT (Internet of Things) used to enhance good agricultural practices. Robotics can be efficiently used in food safety and makes it environment-friendly by using the appropriate use of chemicals. Robotics is also helpful in testing land quality and to choose the appropriate crop for the land. The robotic weed control system is highly beneficial. Development of reconfigurable robot is very important because in the future agricultural land decreases and multitasking robots are required to make it fast and maintain quality, present robots are single task targeted robots. The smart farming also helps to maintain the humidity, temperature and irrigation process. The main aim of this study to making agriculture smart and efficient by applying automation and IoT techniques.
\end{abstract}

Keywords: Automation, IoT, Robotics

\section{INTRODUCTION}

The contribution of agriculture plays an important role in the economic growth of India. In India, more than $60 \%$ of peoples depend upon agriculture. This $60 \%$ of people produce food for the country. Recently, the number of farmers in India decreasing day by day. The agriculture results depend up on the various controlled and uncontrolled factors like sudden change in climatic conditions, high and low rain fall and also the timing of the rain, storms and heat waves, and effects of cold, etc. For these conditions, traditional farming techniques are not appropriate. To minimize these effects and increase the productivity of agriculture, automation and IoT techniques are required. By the help of Automation and IoT, we can measure the environmental condition and water management because these are the most important factors in agriculture [1], [2].

\section{LITERATURE REVIEW}

This paper also deals with how the robotics can be used efficiently used to make good agricultural practices. Automation can be effectively used in food safety, to reduce inappropriate use of chemicals, to protect the environment, to maintain occupational health and in animal welfare. The

Revised Manuscript Received on July 10, 2019 Vidyavardhaka college of engineering Mysuru, Karnataka, India. (Email:

Bharath Y Kashyap, Department of mechanical engineering, Vidyavardhaka college of engineering Mysuru, Karnataka, India (Email: bharath121100@gmail.com)

Guruprasad K, Department of mechanical engineering, Vidyavardhaka college of engineering Mysuru, Karnataka, India (Email: gurusimha14@gmail.com)

Shrinivasa D, Department of mechanical engineering, Vidyavardhaka college of engineering Mysuru, Karnataka India (Email: shrinivasashetty@vvce.ac.in)

Prashant Kumar Shrivastava, Department of mechanical engineering, Vidyavardhaka college of engineering Mysuru, Karnataka, India (Email: er.prashant1986@gmail.com)
Chidananda Kashyap, Department of mechanical engineering, Chidanandakashyap@gmail.com)

record of treatment and steps carried out during the production is needed so GPS is attached to machinery and origin of crop is also obtained. Automation is also used to find soil organic matter, moisture content in it and nutrient availability in the soil. Portable sensors can be used for measurement of several specific locations for agriculture. The robotic weed control system is used to identify weed. Recognition of diseases and pests is made by microbial sensing technology. Automatic record of the condition of crop is obtained by automated machines which can be viewed in different locations by using the software. By this, we can make environment-friendly agriculture[3].

Moreover, we have also studied about the design of a task-based modular reconfigurable agriculture robot. The population in the future grows at a faster rate. There might be a shortage of food and agricultural land also decreases. People prefer to work in comfortable jobs rather doing agriculture. So the solution to this problem is using robotic manipulators. In today's technology, we have single task targeted robots. Creating a universal robot by integrating all the capability to a robot is expensive and complicated. To make different tasks a robot should be reconfigurable. For example, the harvesting task requires both accuracy and large workspace. 3 degrees of freedom i.e., one prismatic and two revolute models can be used to harvest both peach and tangerine. By adding prismatic joints and allowing the order of the revolute and prismatic joints together we can increase the flexibility of the robot. Another approach, multitask problem can be optimized by using different robots for different tasks by using the same software. RRP model takes $15 \%$ less time compared to PRR. RPR is $70 \%$ efficient, RRP is $80 \%-90 \%$ efficient but takes double the time of RPR for harvesting. The proposed method can improve agricultural efficiency and versatility and it is economical [4].

Thereafter, we have discussed about the machine vision applications in agriculture is mainly three type's nondestructive measurements, visual navigation, and behavioral surveillance. "Fixed forward field of view" is important in Machine vision system. There are three FOV, far FOV, near FOV, lateral FOV. FOV gives accurate guidance for agricultural robots. Fixed looking cameras have limitations. FOV of high pixels is used in row guidance. Instead of using one type of FOV, all three types of FOV is combined and variable FOV is made so that three types of views can be obtained in one robot. Variable FOV camera arrangement was used to guide an agricultural robotic platform which can

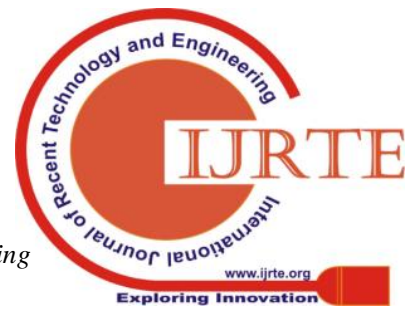


be viewed using a laptop to drive wheels. Machine vision based row guidance is to enable stable navigation of robot, distinguish green corn plants and soil background. Size of the robot is optimal. GPS is attached to the robot to find location. Morphological operations were used to calculate guidance lines in the field. Far FOV is more efficient compared to near FOV [5].

Moreover, in Japan rapid depopulation of farming communities is taking place. The solution for this is to use farming robots to perform farm operation accurately at the right time. It allows one operator to control multiple vehicles, the goal is to get fully robotized operation in rice production. Development of three types of the robot is done. First is Robot tractor which performs tillage and puddling. Secondly, rice planter robot which performs transplanting, fertilizing and herbicide application and thirdly combines harvester robot. Robot tractor has controlled steering by stepping motor, it can be put into manual operator mode and GPS is attached to it to know its location and can be operated using computers. Many sensors are present in it for the safety of men and obstacle sensors indicates obstacles by a warning sound and warning light. This results in Robot farming system into the labor of a person in Japan [6]. Moreover, the robotic tractor and combine harvester came into existence in Japan. But for heavy weight crops existing technology is not suitable. Hence the robotic arm, end effector and control algorithm was designed. The robotic arm can be used for crop sampling and field monitoring. The harvesting robot is composed of a robot tractor, robot arm, end-effector, positioning system, vision system, and a controlling unit. The robotic arm is powered by a servo motor. It has five joints and is capable of withstanding payload of $200 \mathrm{~N}$ with FOS- 2. Forward and Inverse kinematics are used for getting joint parameters such as joint angle, velocity and acceleration time. Using the parameters the robotic arm was designed for minimum vibration and maximum efficiency. The accuracy and repeatability are enough for the harvesting of heavy weight crops and can also be used for spraying using different end-effectors [7].

Here we deal with the ASETA project. It is the one which deals with the ground-based and aerial-based vehicles to identify and localize the weeds in the field. This project is only constrained within the first two levels of treatment of the field, i.e. individual plant treatment and treatment of grids. This ASETA project works with the current state of the field and the treatment to be applied to gain the immediate good yield. There are many more projects like RHEA. RHEA project is similar to ASETA i.e. to minimize the usage of pesticides. This kind of projects will enhance or increase the knowledge over the fields of robotics, agriculture and the vision of artificial intelligence. The main idea of the ASETA project is to have a multiscale imaging approach i.e. to take the aerial images from high altitude and lowering it gradually to the lower altitude for the purpose of obtaining the greater resolution images. The next stage will be of the system architecture which is based on the proper designing of the vehicle. Then comes the stage of automatic planning which is required as it reduces the human effort every time. After this stage, the path planning is to be done. It is necessary to design a specific path for the vehicle so that it should not destroy any kind of crops while moving in the field. Then comes the aerial image processing, it is the process which is mentioned earlier here i.e. the image capturing. Next stage is of ground vision. As just the aerial image is not the complete source for the solution, we need ground vision also. Then the last stage i.e. cooperation, all the information gathered should go in cooperation so that we can achieve the best results [8].

We are living in a world which is ever growing. But because of this growth we face some problems also, one such kind of problem is the food scarcity. In order to stop this, we have to find new kind of techniques to increase food production. Researchers are always on this work. Now Japan is trying to overcome this problem by using the multiple robot tractors. This process consists of many stages. Firstly, the robot platforms. The laboratory of vehicle robotics has developed an electronic robot and two robot tractors. These can be very useful as they can function on their own like going forward, backward, speed steering, PTO, etc. Next comes the stage of sensors. In that first comes the navigation sensors. They use many GPS trackers like hemisphere GPS trackers and AGI-3 GPS trackers to choose the path which they should follow. Then comes the safety system of sensors. Here the sensors like twodimensional laser scanners and many more are used to find out the obstacles present in their way and to change their movement. This kind of system reduces the human effort in the field setting and they can concentrate on improving the production in other ways [9].

Thereafter, many researchers are trying to introduce the robotics or mechatronics to the field of agriculture. Such kind of thing is the agricultural mobile robot. This mobile robot is designed to sense the agronomic parameters or the importance of the most important crop kinds. When designing these kinds of robots we have to make it for a universal approach to handle the crops even from one foot to even six-foot ones like jute also. In order to make this we have to take care of the diameter and frame width of the working tool primarily. Then comes the traction. It is the system which is commonly used to distribute the loads on the soil in order to reduce the compacting and disturbing of it. This can be mainly seen in the wheels and tracks. Next comes the steering part. This is one of the important components, as it is necessary to manage the rover's direction of movement. There are many types in this like differential steering, articulated steering, and wheel steering. Next, come the motors and power supply which are the important components to run the robot. Then comes the electronic control. Next is the mechanical structure which is the most important one as it should be as friendly as possible so that agriculture should not be disturbed [10].

Moreover, to develop the field of agriculture we have to work efficiently. People are trying to implement many changes in it. One such effort is field phenotyping robots. To construct any kind of robots, the structure is the basic designing thing. Here also structure is very important as it should work in the fields without disturbing the existing crops. It is a system which moves in a beam kind of support

Published By: 
with a clear vision system. It clearly identifies the spot of planting and it will do the planting process with precision. The workflow of the system should be very important here. It should know the task to be performed. It should know the exact place and position of work. The height of working manipulator should be proper. As this is mostly used in the rice fields it should clearly understand the rice separating process and act on it properly. The other parameters which are needed to be taken care of are the length of arms, the height of equipment's [11].

One such thing is the pollination of seeds. When insects go on the flowers for their food or the energy source, the seeds which will be present in the flower will get attached to the insects then it travels and sits on the other flower of having opposite gamete followed by fertilization. This process is called pollination. But people are trying to incorporate the robotic technology into this field. Introducing the robotic bees is not the best solution we have right now. The using of the robotic things into this field can result in an economic loss as these things cannot be done by one single robot and we require n number of them. Each one will cost a huge value. One small mistake done by the robots can result in the unacceptable high environmental cost. The ecosystem can also be damaged by this kind of things as they are made of non-degradable or in other sense they cannot be handled by nature to get things done right. By this, it can also affect biodiversity in many ways. These can not only affect the nature, but it can also affect humans by causing the food insecurity [12].

\section{RESULT AND DISCUSSION}

The traditional farming process based up on humans. From the starting of the farming process to end process needs the human-like Ploughing, Spray seeds, Fertilizer, check the water level and availability and Harvesting.

In smart farming, sensors are applied for gathering the data from various filed like temperature sensor, Humidity sensor, Soil moisture sensor, Think to speak IoT app, Water motor, and sprinklers. These sensors are well connected with the microcontroller. Microcontroller received the data from the sensors and check the threshold values. Then according to the threshold values, it takes the needful action and also informed the farmer by the message. The all the process completed by the $\mathrm{Wi}-\mathrm{Fi}$ module. Nowadays, the culture of the poly house increasing day by day because in the poly house setup we can control the temperature, humidity, pest attack, and irrigation process. By smart farming, we can also minimize the human efforts to maximize productivity[13][14].

\section{CONCLUSIONS}

The objective of this study to increase productivity and minimize time and human efforts. By applying the automation and smart farming techniques we can change the action according to the crop, weather conditions, and soil, etc.

1. In the field of irrigation automation and smart systems can irrigate the horticulture land, parks, gardens, and golf courses efficiently and reduce the wastage of water.
2. The uses of automation and smart systems also help in food safety by the selection of appropriate chemicals.

3. The weed control system is also benefited by these technologies.

4. The application of multitasking robots is also useful to make it fast and maintain quality.

5. In the smart farming also helps to maintain the humidity, temperature and irrigation process by using the sensors and apps.

\section{ACKNOWLEDGEMENT}

The manuscript is prepared by taking assistance from Accendere Knowledge Management Services Pvt. Ltd. We are thankful to them. We also express our gratitude to our teachers and mentor for guiding us throughout the work.

\section{REFERENCES}

1. V. Sirisha and D. Somasekhar, "Design and development of Agriculture System Based on IoT,” 2019.

2. K. Lakhwani, H. Gianey, N. Agarwal, and S. Gupta, "Development of IoT for Smart Agriculture a Review," Springer, Singapore, 2019, pp. 425-432.

3. J. De Baerdemaeker, "Precision agriculture technology and robotics for good agricultural practices," IFAC Proc. Elsevier, 2013.

4. M. Levin, A. D.- IFAC-PapersOnLine, and U. 2016, "Design of a Task-Based Modular Re-Configurable Agricultural Robot," IFAC-PapersOnLine, vol. 49, no. Issue 16, p. Pages 184-189, 2016

5. J. Xue, L. Zhang, T. G.-C. and E. in Agriculture, and U. 2012, "Variable field-of-view machine vision based row guidance of an agricultural robot," Comput. Electron. Agric., vol. Volume 84, p. Pages 85-91, 2012.

6. K. Tamaki, Y. Nagasaka, K. Nishiwaki, ... M. S.-I. P., and U. 2013, "A robot system for paddy field farming in Japan," IFAC Proc. Vol., vol. 46, no. Issue 18, p. Pages 143-147, 2013.

7. T. Kamata, A. Roshanianfard, N. N.- IFAC-PapersOnLine, and U. 2018, "Heavy-weight Crop Harvesting RobotControlling Algorithm," IFAC-PapersOnLine, vol. 51, no. Issue 17, p. Pages 244-249, 2018.

8. K. Hansen, F. Garcia-Ruiz, ... W. K.-I. P., and U. 2013, "An autonomous robotic system for mapping weeds in fields," IFAC Proc. Vol., vol. Volume 46, no. Issue 10, p. Pages 217 224, 2013

9. N. Noguchi, O. B. J.-I. P. Volumes, and U. 2011, "Robot farming system using multiple robot tractors in Japan agriculture," IFAC Proc. Vol., vol. Volume 44, no. Issue 1, p. Pages 633-637, 2011.

10. R. Tabile, E. Godoy, ... R. P.-I. P., and U. 2010, "Design of the mechatronic architecture of an agricultural mobile robot," IFAC Proc., vol. Volume 43, no. Issue 18, p. Pages 717-724, 2010.

11. J. Zhang et al., "Field phenotyping robot design and validation for the crop breeding," IFAC-PapersOnLine, vol. Volume 49, no. Issue 16, p. Pages 281-286, 2016.

12. S. Potts, P. Neumann, ... B. V.-S. of the total, and U. 2018, "Robotic bees for crop pollination: Why drones cannot replace biodiversity," Sci. Total Environ. , vol. Volume 642 p. Pages 665-667, 2018. 
13. K. Sneha, R. Kamath, M. Balachandra, and S. Prabhu, "New Gossiping Protocol for Routing Data in Sensor Networks for Precision Agriculture," Springer, Singapore, 2019, pp. 139152.

14. L. Kamelia, M. A. Ramdhani, A. Faroqi, and V. Rifadiapriyana, "Implementation of Automation System for Humidity Monitoring and Irrigation System," IOP Conf. Ser. Mater. Sci. Eng., vol. 288, no. 1, p. 012092, Jan. 2018. 